\title{
Pre-operative fibrinogen levels are predictive of blood loss in total hip replacement surgery
}

Pedrosa, Filipa; Marques da Costa, Filipe; Resende, Alexandra

Serviço de Anestesiologia - Prof. Dr. Lucindo Ormonde

\section{Introduction}

- Fibrinogen is considered an essential part of the clotting cascade, crucial for the formation of the fibrin polymer.

- Studies have shown that low levels of fibrinogen are associated with increased blood loss and PRBC units transfused during surgery.

- In major surgery a rapid decrease of fibrinogen levels due to consumption is expected.

Improving pre-operative fibrinogen levels can potentially reduce blood loss and the consumption of blood products.

\section{Methods}

- Retrospective trial

- Hemotherapeutic practice in elective total hip replacement (THR)

- 82 patients

- Preoperative fibrinogen correlated with hemoglobin variation, post-operative hemoglobin in the first 48 hours, PRBC units transfused.

\section{Results}

- Lower pre-operative fibrinogen levels were found in older patients ( $>65$ years-old) $(p=0,05)$.

- Lower pre-operative fibrinogen levels were found in patients with a higher ASA classification $(p=0,002)$.

- A positive correlation was found between fibrinogen levels $<300 \mathrm{mg} / \mathrm{dL}$ and the hemoglobin variation $(p=0,022)$.

\begin{tabular}{|c|c|c|c|c|c|}
\hline & $\mathbf{N}$ & MIN. & MAX. & MEAN & $\begin{array}{l}\text { STD. } \\
\text { DEV. }\end{array}$ \\
\hline SEX & $82(100 \%)$ & - & - & - & - \\
\hline Male & $45(54,2 \%)$ & - & - & - & - \\
\hline Female & $37(44,6 \%)$ & - & - & - & - \\
\hline AGE & $82(100 \%)$ & 25 & 85 & 64,35 & 13,383 \\
\hline ASA & $82(100 \%)$ & - & - & - & - \\
\hline I & $7(8,4 \%)$ & - & - & - & - \\
\hline II & $56(67,5 \%)$ & - & - & - & - \\
\hline II & $19(22,9 \%)$ & - & - & - & - \\
\hline ANESTHESIA & $82(100 \%)$ & - & - & - & - \\
\hline General Anesthesia & $22(26,5 \%)$ & - & - & - & - \\
\hline Regional Anesthesia & $42(50,6 \%)$ & - & - & - & - \\
\hline Combined Anesthesia & $6(7,2 \%)$ & - & - & - & - \\
\hline Missing & $12(15,7 \%)$ & - & - & - & - \\
\hline PRE-OP. FIBRINOGEN & $82(100 \%)$ & 130 & 686 & 321,98 & 82,33 \\
\hline$<200 \mathrm{mg} / \mathrm{dL}$ & $3(3,6 \%)$ & - & - & - & - \\
\hline $201-300 \mathrm{mg} / \mathrm{dL}$ & $31(37,3 \%)$ & - & - & - & - \\
\hline $301-400 \mathrm{mg} / \mathrm{dL}$ & $41(49,4 \%)$ & - & - & - & - \\
\hline$>400 \mathrm{mg} / \mathrm{dL}$ & $7(8,4 \%)$ & - & - & - & - \\
\hline \multicolumn{6}{|l|}{ HEMOGLOBIN } \\
\hline PRE-OP. Hb & $82(100 \%)$ & 7,9 & 17,7 & 13,46 & 1,83 \\
\hline POS-OP. Hb & $82(100 \%)$ & 6,1 & 14,2 & 10,68 & 1,67 \\
\hline Hb D2 & $82(100 \%)$ & 7,3 & 13,2 & 9,98 & 1,39 \\
\hline$\Delta H b$ & $82(100 \%)$ & $-1,9$ & 8,6 & 3,74 & 1,68 \\
\hline PRBC Transfused & $41(50 \%)$ & 0 & 10 & 1,8 & 1,928 \\
\hline
\end{tabular}

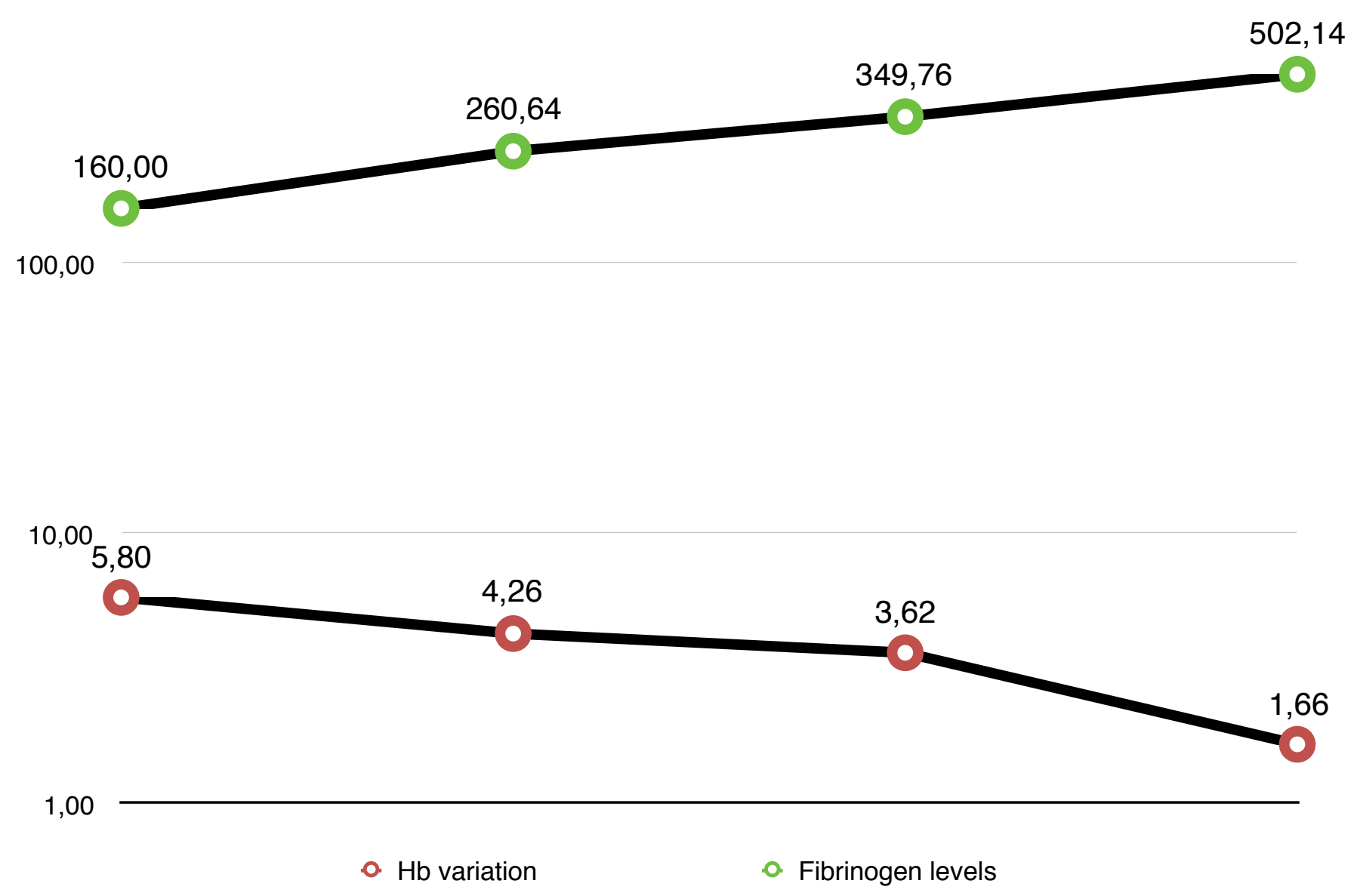

\section{Conclusion}

- Older patients or patients with higher ASA physical status classification might be at risk of having lower fibrinogen.

- Routine pre-operative dosing of fibrinogen in these patients might be valuable.

- In THR surgery a higher fibrinogen threshold might be adequate to reduce peri-operative blood loss.

- Implementation of protocols regarding pre-operative blood management can improve outcomes by reducing both blood loss and the need for transfusion. 